\title{
Conciliation emploi-famille et horaires chez les paramédics (ambulanciers): des différences selon l'âge et le genre
}

\author{
LAZZARI DODELER, Nadia et TREMBLAY, Diane Gabrielle \\ Téluq-Uqam (Montréal, Canada) \\ mails : ndodeler@hotmail.com \\ dgtrembl@teluq.ca
}

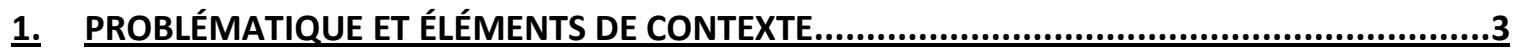

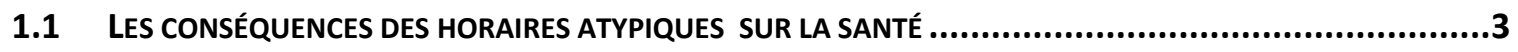

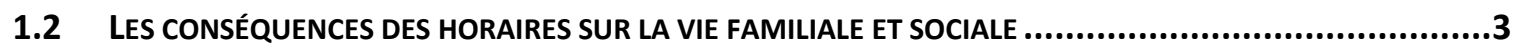

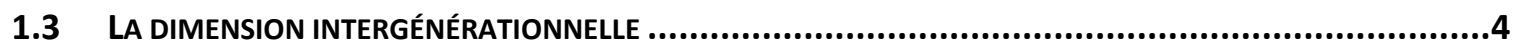

1.4 LES CONFLITS ENTRE LE TRAVAIL ET LA VIE PERSONNELLE DES EMPLOYÉS ÂGÉS................................4

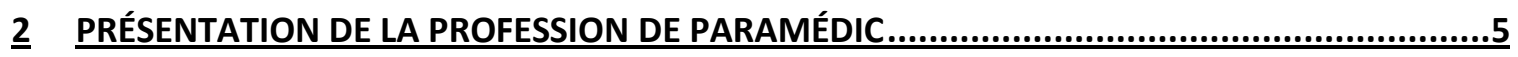

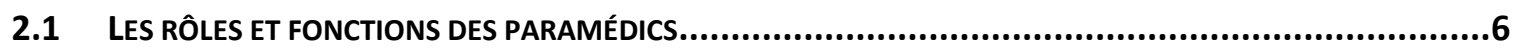

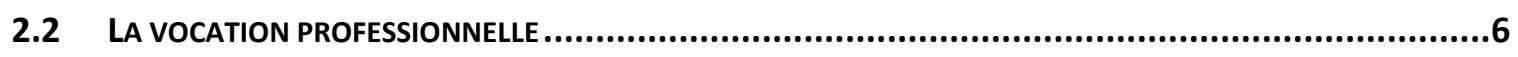

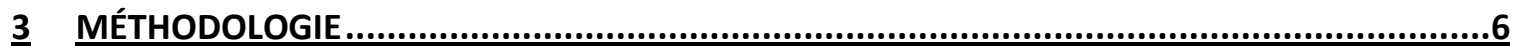

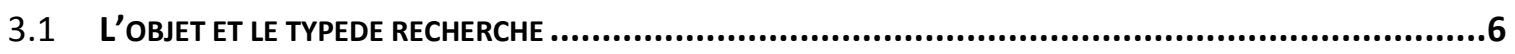

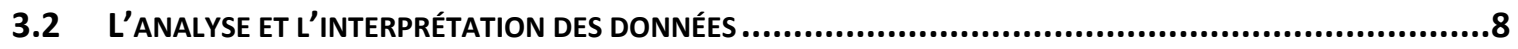

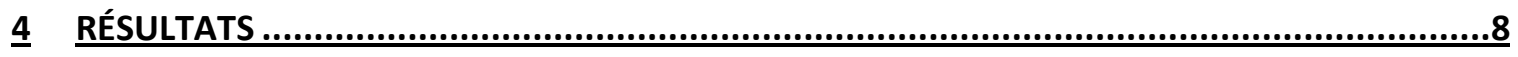

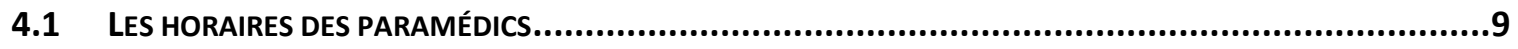

4.1.1 IMPACT DES HORAIRES SUR LE STRESS, LE SOMMEIL ET L'IRRITABILITÉ ......................................... 9

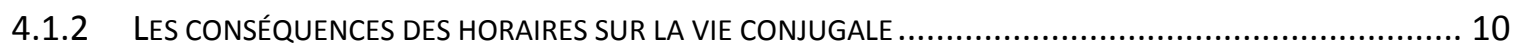




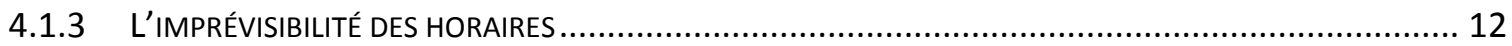

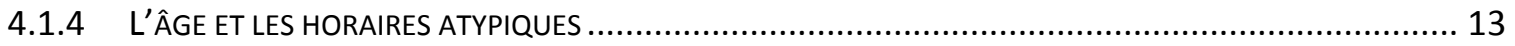

4.2 LES PRATIQUES D'ARTT DU POINT DE VUE DE LA CONCILIATION TRAVAIL-FAMILLE..........................13

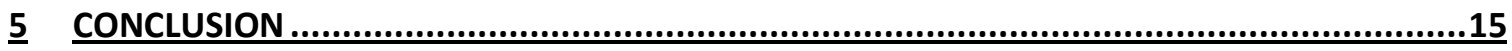

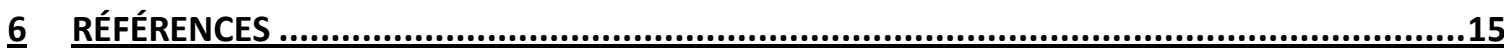

\title{
Résumé
}

Certains emplois ont du mal à offrir un environnement permettant une bonne articulation entre la sphère professionnelle et la sphère familiale. Ces emplois exigent des employés prêts à répondre à un appel à n'importe quelle heure du jour ou de la nuit. C'est le cas des paramédics (ambulanciers) au Québec. Nous avons voulu savoir si, selon leur sexe et leur âge, ces employés vivent de la même manière les horaires atypiques et si cela a une incidence sur la conciliation emploi-famille.

\section{Mots-clés}

Gestion des âges, classe d'âge, conciliation emploi-famille, para-médical, GRH.

\begin{abstract}
Some jobs do not provide an ideal environment for a good articulation between the professional and the domestic sphere. These jobs require employees ready to answer a call at any time of day or night. This is the case of paramedics (ambulance) in Quebec. We analysed the impact of these unsocial schedules, by gender and age, and impact on their work-family balance
\end{abstract}

\section{Keywords}

Age management, age group, work-family balance, paramedics, HRM 


\section{Problématique et éléments de contexte}

Les mutations du marché du travail, de la famille, et les changements démographiques occasionnent des difficultés pour concilier le travail et la vie personnelle au Québec comme ailleurs. (Duxbury et al., 1994; Tremblay, 2008, 2012 ).De plus, certains secteurs d'activité connaissent des difficultés encore plus importantes, en raison d'horaires dits atypiques : des horaires de 24 heures par jour, 7 jours par semaine (Lazzari et Tremblay, 2010);c'est le cas chez les ambulanciers. Dans des secteurs comme la santé ou le secteur policier, ces horaires sont également fréquents, en raison de l'obligation de service à la population. (Tremblay et Larivière, 2010; Tremblay, 2012b). L'Amérique du Nord compte 20 à $25 \%$ de salariés travaillant selon ce type d'horaire et environ un tiers d'emplois atypiques (Tremblay, 2004), soitle travail de soir ou de nuit, de fins de semaine, sur appel, temps supplémentaire, quarts de travail, semaines comprimées, horaires variables imprévisibles, horaires brisés etc. (Tremblay, 2012a,b; Presser, 2004; Shields, 2000). Ces secteurs d'emploi étant moins étudiés en lien avec la conciliation emploi-famille, nous avons choisi de nous y intéresser, car outre les problèmes de conciliation, il y a aussi des incidences sur la santé.

\subsection{Les conséquences des horaires atypiques sur la santé}

Qu'il s'agisse de jeunes employés ou de plus âgés, les horaires atypiques ont une incidence sur la santé (Morin, 2008; Tremblay, 2012b). Lutter contre son horloge interne en travaillant sur des quarts de travail la nuit peut entraîner des troubles gastro-intestinaux, un manque de sommeil, une altération du jugement et de la prise de décisions (Desjardins, 2004, Morin, 2009). En ce qui a trait aux ambulanciers, les analyses de la Commission de la santé et sécurité au Travail(Corbeil \& al.,2010) révèlent que près de $60 \%$ des accidents du travail chez les techniciens ambulanciers sont liés à un effort excessif (soulever, tirer, etc.) ou une réaction du corps (se pencher, glisser etc.). Les employés qui ne réussissent pas à concilier leur travail et leur vie personnelle sont plus stressés, peu satisfaits et moins engagés vis-à-vis de l'organisation (Duxbury et al., 1994). Cela se traduit par de l'absentéisme, des retards, de la démotivation, de la rotation de personnel, des difficultés pour attirer et retenir les bons employés. (Tremblay, 2012a ; Paris, 1989; MacBride, 1999). Ainsi en l'absence de programmes explicites de conciliation travail-famille, certains employés peuvent vivre des difficultés importantes liés aux horaires et cela peut avoir des incidences sur la santé, la vie familiale et sociale et parfois donner envie de quitter ce type d'emploi.

\subsection{Les conséquences des horaires sur la vie familiale et sociale}

Des recherches récentes permettent de mettre en évidence les difficultés d'articulation des temps professionnels, familiaux et sociaux. David (2007) parle de désynchronisation des temps sociaux, collectifs ou individuels. Presser (2004) et David (2007) observent la raréfaction des temps de qualité entre les parents et les enfants en ce qui concerne la prise des repas, la sociabilité familiale, les loisirs collectifs. D'autres recherches (Pailhé \& Solaz, 2010) soulignent qu'en terme de trajectoire professionnelle, l'arrivée d'un enfant peut marquer une rupture chez les jeunes femmes, parfois un renoncement à l'activité professionnelle pour d'autres. Les tensions entre la vie familiale et les responsabilités 
professionnelles peuvent également avoir un effet négatif sur la vie conjugale (Allen \& coll., 2000). De même, selon Presser (2004), les horaires atypiques (soir, nuit, week-end) réduisent la qualité du temps familial. S'appuyant sur des enquêtes menées aux ÉtatsUnis, l'auteur a montré que les conflits dus à ce type d'horaires étaient plus fréquents au sein des couples et pouvaient aboutir à une fragilisation de ce dernier. Nous concluons donc que les horaires atypiques engendrent des difficultés sur le plan de la santé, de la conciliation et de la vie familiale et sociale. Les travaux font aussi état d'effets différenciés sur la santé physique et l'apprentissage selon l'âge, comme le montre la section suivante.

\subsection{La dimension intergénérationnelle}

Actuellement, quatre générations (la génération du Baby-Boom, celle des $\mathrm{X}$, des $\mathrm{Y}$ et maintenant des $\mathrm{C}$, fortement axée sur les outils de communication) se côtoient sur le marché du travail. Toutes ont eu à faire face aux mêmes défis d'insertion sur le marché du travail, de développement de carrière puis finalement de retraite (Gavrancic et al, 2009). Par contre, l'âge peut jouer différemment selon les exigences du travail (Tremblay, 2012b). Dans certains domaines de travail de nature plus physique, les plus âgés peuvent se trouver en difficulté, alors qu'au contraire dans les secteurs où l'expérience et les connaissances accumulées sont importantes, ils peuvent être favorisés. Marbot (2007) attire l'attention sur le fait que la pédagogie et le mode d'apprentissage en formation continue doivent également être adaptés aux besoins des individus pour ne défavoriser aucune classe d'âge. Les recherches réalisées dans le secteur de la santé témoignent des difficultés propres à ce milieu avec l'avancée en âge, celles-ci étant liées aux exigences physiques fortes de certains postes, à la fatigue professionnelle accumulée et à la nécessité de s'adapter sans cesse à de nouvelles techniques de travail. (David, 2007; Cloutier et al., 2007). Se pose alors fortement la question de la pénibilité du travail dans la santé (Anact, 2012). Pour attirer et conserver leur personnel, les organisations devraient développer des pratiques de gestion en lien avec les attentes et les caractéristiques personnelles liées aux diverses générations présentes sur le marché du travail (Tremblay, 2010, 2007, dir). Alors que les plus âgés recherchent davantage la stabilité, le revenu, un bon régime de retraite, les plus jeunes rechercheraient les défis, des mesures de conciliation mais aussi des cadres qui adoptent des attitudes de mentor ou de coach (Tremblay, 2010).

\subsection{Les conflits entre le travail et la vie personnelle des employés âgés}

Alors qu'on s'est beaucoup intéressé à la conciliation emploi-famille chez les parents de jeunes enfants (Tremblay, 2012a,b), peu de recherches ont été effectuées chez des employés âgés, alors que la conciliation pose des défis tout au long du parcours de vie, et peut avoir des incidences sur les décisions liées à la retraite (Uriarte-Landa et Hébert, 2009). Au Canada plus de $25 \%$ de retraités auraient continué à travailler s'ils avaient pu bénéficier d'horaires allégés sans perte financière, $27 \%$ si leur santé avait été meilleure et $12 \%$ s'ils n'avaient pas été obligés de prendre leur retraite par obligation (Morissette, Schellenberg et Silver, 2004). Une autre étude canadienne a examiné l'insatisfaction des travailleurs âgés de 55 ans et plus, en lien avec l'équilibre travail-vie-personnelle. Cette étude souligne que $14 \%$ des personnes de ce groupe d'âge étaient insatisfaites de leur articulation travail-vie-personnelle comparativement à $25 \%$ pour le groupe des $25-54$ ans. La principale raison invoquée était la surcharge de travail (45\%), suivie du manque de temps à consacrer à la famille ( $27 \%$ contre $46 \%$ pour les $25-44$ ans) (Uriarte-Landa et 
Hébert, 2009). Pour contrer l'exclusion de ces travailleurs, il pourrait donc être intéressant de leur permettre de réaménager leurs horaires, de leur proposer d'autres tâches en lien avec leur âge et leur état de santé, une hypothèse que nous avons voulu tester auprès du groupe des paramédics, puisqu'elle avait été validée dans d'autres travaux (Tremblay et Genin, 2010). Outre les infirmières (Tremblay et Larivière, 2010), sur lesquelles des travaux ont été réalisés, un des groupes professionnels qui semble présenter le plus de difficultés à concilier le travail et la famille serait celui des paramédics. Nous nous sommes donc intéressées à cette profession pour mieux comprendre les enjeux liés à la conciliation emploi-famille dans une perspective de parcours de vie, mais présentons cette profession avant d'aller plus loin.

\section{Présentation de la profession de paramédic}

Selon Service Canada (2009), les paramédics ou ambulanciers sont 3234 au Québec et cela regroupe $66,9 \%$ de personnes situées dans la tranche d'âge de $15-44$ ans et $33,1 \%$ situées dans celle des 45-65 ans et plus. Il s'agit donc d'un domaine d'emploi où les gens sont relativement jeunes, $57,9 \%$ se situant dans le groupe des $25-44$ ans, soit celui le plus touché par les problématiques de la conciliation travail-famille. Jusqu'en 1991, les femmes ne représentaient que $16 \%$ des travailleurs de la profession de paramédic (Services Canada, 2009). Ce n'est qu'à compter des années 2006 qu'on observe une progression de ces dernières dans le secteur ambulancier, alors qu'elles occupent $23,4 \%$ des postes, 76, 6\% étant occupés par des hommes (Services Canada, 2009).

Il aurait été intéressant d'obtenir des statistiques officielles sur les taux d'emploi à temps partiel des 20-44 ans et des 45-60 ans et plus, tranches d'âge que nous utilisons dans nos analyses, mais les seules données disponibles correspondent à la tranche d'âge des 20-39 ans, qui représente $10,19 \%$ des effectifs (MSSS, 2002). De plus, en ce qui concerne les départs à la retraite, les personnes âgées de 55 ans et plus représentent $4,33 \%$ de l'effectif et il est prévu qu'ils partiront à la retraite dans les dix prochaines années. (MSSS, 2002)

La croissance de la demande de transport, les longs horaires, la rotation élevée du personnel, un taux d'absentéisme élevé ( $30 \%$ des heures travaillées), l'abandon pour certains de la profession, ainsi que les départs à la retraite font que la rétention en emploi des paramédics semble difficile (Emploi Québec, 2003; Service Canada, 2010). Toutes ces difficultés ont engendré dans de nombreuses régions du Québec (semi-urbaines et rurales) un délai de réponse aux appels de 2 à 4 fois supérieur à celui des grands centres (CSN, 2006). Ceci est dû au fait que dans les grands centres, les paramédics sont en attente dans les véhicules et qu'ils travaillent sur des horaires à l'heure (8-9-10-12 heures/jour) alors que de nombreux paramédics travaillant dans les régions semi urbaines et rurales sont en attente à la maison et travaillent sur des horaires de faction de 7/14 (7jours de travail $24 \mathrm{~h} / 24$, puis 7 jours de congé) (CSN, 2006).

A la lumière de ces éléments, comme de nos entretiens, il faudrait améliorer les conditions de travail afin que d'une part, les plus jeunes ne migrent plus vers les grandes villes ou ne quittent pas le métier prématurément, et que d'autre part, les plus âgés puissent demeurer plus longtemps en emploi, s'ils le souhaitent. C'est du moins dans ce sens que vont les recommandations de nombreux travaux visant à attirer la main-d'œuvre ayant à concilier 
emploi et famille (Tremblay, $2012 \mathrm{a}, \mathrm{b}$ ) et à retenir pour prolonger les années de travail des plus âgés (Tremblay, 2010, dir., 2007).

\subsection{Les rôles et fonctions des paramédics}

Au Canada et au Québec, le terme de «paramédic» qualifie les praticiens qui travaillent dans les services ambulanciers. Il renvoie à la fonction de soins et non seulement à la personne qui conduit une ambulance, cette dernière étant connue sous le nom de technicien ambulancier (APPQ, s.d.). Ainsi, le paramédic désigne celui qui pose des soins paramédicaux. Lors de leurs interventions, les paramédics doivent questionner leurs patients afin d'entreprendre les soins de stabilisation en lien avec leur état de santé. Ces soins incluent la ventilation avec un ballon-masque, la défibrillation semi-automatique, l'installation d'un tube (Combitube (C) pour sécuriser les voies respiratoires, de même que l'administration de certains médicaments par voies intramusculaires, sous-cutanée, souslingual, orale, intra-nasale, et par inhalation. Pour ce faire, ils suivent des protocoles d'interventions préhospitaliers validés par les médecins en poste en tant que directeurs médicaux régionaux (APPQ, s.d.).

\subsection{La vocation professionnelle}

Selon Chevandier (2009), alors que le terme «vocation» était intimement lié à l'histoire religieuse, depuis le XIXème siècle le terme «vocation» est utilisé dans les domaines de l'art et de la littérature et c'est à partir de la seconde moitié du XXème siècle qu'il apparaît dans le registre du travail, de la profession. Ainsi, dans certains cas, on ferait le choix d'une profession comme on entrerait en religion. Weber (1918) évoque également l'idée de vocation professionnelle, accordant une place centrale au «Beruf», terme intégrant l'idée de vocation et de profession. Selon l'auteur, l'aliénation du travail, en l'absence de ferveur, ne permet pas au travailleur de discerner l'intérêt que procure le fruit de son activité. En effet, la vocation ne tient pas qu'aux conditions extérieures du travail mais aussi aux dispositions intérieures de l'individu (Weber 1918). Les métiers dits «de vocation» sont souvent ceux dont les dimensions d'aide à autrui et de dévouement à une cause, sont très présentes (Fusulier et al, 2009), comme c'est le cas chez les ambulanciers. Lalive d'Epinay 1994, cité dans Fusulier \& al. (2008) précise que la tension travail-famille est plus importante lorsque l'ethos du devoir (la collectivité est un principe supérieur à l'individu) se désagrège au profit d'un ethos de l'épanouissement personnel. Dans un tel contexte, l'idée de sacrifier sa vie personnelle au profit de sa vie professionnelle s'accepte plus difficilement. Cela semble être plus souvent le cas pour la nouvelle génération d'employés, qui hésite à sacrifier leur vie personnelle ou familiale.

\section{Méthodologie}

\subsection{L'objet et le type de recherche}

Dans le but de préparer la négociation de leur convention collective, un des syndicats de paramédics nous a demandé d'examiner les difficultés engendrées par les horaires atypiques des paramédics (vivant dans une zone semi-urbaine), en lien avec la conciliation travail-famille. Cette étude est particulièrement intéressante parce qu'elle s'intéresse à un 
secteur majoritairement masculin, alors que l'on s'intéresse plus souvent aux femmes dans la santé (Tremblay, 2012b), et aussi parce que nous tenons compte de Ainsi notre recherche avait pour but de produire une étude des perceptions et de la diversité des situations en fonction de l'âge et du genre.

Nous avons fait le choix d'une approche inductive d'analyse fondée surtout sur des données qualitatives (Thomas, 2006), mais également sur des données quantitatives, bien que le nombre réduit de répondants ne nous autorise pas à faire des analyses statistiques très poussées. Notre objectif était de connaitre les pratiques mises en œuvre, et de tenter de comprendre si les mesures de conciliation existantes peuvent avoir des effets - positifs ou négatifs - sur la rétention de la main-d'œuvre, selon l'âge et le genre. En novembre 2010, nous donc avons effectué une enquête auprès des paramédics québécois en lien avec leurs horaires de travail,la conciliation travail-famille, les fins de carrière, ainsi que les effets perçus des horaires sur la santé des employé(e)s.

Nous avions aussi mis de l'avant quelques hypothèses de travail, à savoir que les conséquences des horaires seraient particulièrement difficiles pour les jeunes hommes et femmes ayant de jeunes enfants et que les difficultés se maintiendraient davantage pour les femmes plus âgées, puisqu'elles continuent d'assumer les responsabilités familiales, alors que les hommes tendraient à s'en dégager au fil des ans et qu'ils sont toujours davantage investis dans le milieu de travail que dans la famille. Sans pouvoir conclure de manière définitive, étant donné les nombres limités, nous pourrons tout de même présenter quelques réponses exploratoires.

Le tableau 1 présente les répondants. Les entrevues ont été basées sur un questionnaire développé à partir de nos recherches antérieures (une partie qualitative et la seconde quantitative) sur le thème de la conciliation emploi-famille (Tremblay, 2012a), mais aussi sur les mesures d'attraction et de rétention de la main-d'œuvre (Lazzari-Dodeler et Tremblay, 2010).

\section{Tableau 1. Répartition des répondants selon l'âge et le genre}

\begin{tabular}{|c|c|c|c|c|}
\hline $\begin{array}{l}\text { Date des entrevues ou de } \\
\text { l'envoi du questionnaire }\end{array}$ & Type de questionnaire & Résultats & Homme & Femme \\
\hline $\begin{array}{l}17 \text { au } 18 \text { novembre } 2010: 17 \\
\text { entrevue de } 50 \text { minutes chacun }\end{array}$ & $\begin{array}{l}\text { Grille d'entretien composée } \\
\text { d'une partie qualitative et } \\
\text { quantitative. }\end{array}$ & $\begin{array}{l}17 \text { entrevues : } \\
\text { Dont : } \\
20-44 \text { ans } \\
45-60 \text { ans }\end{array}$ & $\begin{array}{l}6 \\
4\end{array}$ & $\begin{array}{l}6 \\
1\end{array}$ \\
\hline $\begin{array}{l}14 \text { décembre } 2010: \text { envoi } \\
\text { numérique de } 120 \\
\text { questionnaires }\end{array}$ & $\begin{array}{l}\text { Seule la partie quantitative du } \\
\text { questionnaire a été envoyée. }\end{array}$ & $\begin{array}{l}60 \text { réponses : } \\
\text { Dont : } \\
20-44 \text { ans } \\
45-60 \text { ans }\end{array}$ & $\begin{array}{l}27 \\
24\end{array}$ & $\begin{array}{l}8 \\
1\end{array}$ \\
\hline \multicolumn{3}{|c|}{ Total hommes-femmes : } & 61 & 16 \\
\hline \multicolumn{3}{|c|}{ Total } & \multicolumn{2}{|c|}{77} \\
\hline
\end{tabular}


Dans nos statistiques descriptives, nous tenons compte des réponses de l'ensemble des 77 répondants, puisque les personnes interviewées ont aussi répondu à la partie quantitative.

\subsection{L'analyse et l'interprétation des données}

A partir des entrevues, nous avons procédé, dans un premier temps, à une analyse par résumé, entretien par entretien, pour chacun des thèmes figurant dans notre guide d'entretien (Gavard-Perret et al., 2008). Puis, dans un second temps, par une approche horizontale, nous avons relevé les thèmes récurrents dans chaque entretien par le procédé de l'analyse thématique des données, selon nos thèmes et sous-thèmes (Gavard-Perret et al., 2008). Dans cet article, nous dressons d'abord le portrait des paramédics selon l'âge, le genre et le statut d'emploi. Puis nous examinerons les différences vécues selon l'âge et le genre en lien avec les horaires de travail, la conciliation travail-famille et les fins de carrière.

\section{Résultats}

Avant de présenter les résultats, le tableau 2 présente le portrait de nos répondants paramédics en fonction du genre, de l'âge et de leur statut d'emploi, à savoir temps complet et temps partiel.

\section{Tableau 2. Portrait des répondants selon le genre, l'âge et le statut d'emploi}

\begin{tabular}{|l|c|c|c|c|c|c|}
\hline & \multicolumn{2}{|c|}{ Tranche d'âge } & \multicolumn{3}{|c|}{ Temps complet par tranche d'âg } & \multicolumn{2}{|c|}{ Temps partiel par tranche d'âg } \\
\hline Répondants & $20-44$ an & $45-60$ an & $\begin{array}{c}20-44 \\
\text { ans }\end{array}$ & $\begin{array}{c}45-60 \\
\text { ans }\end{array}$ & $\begin{array}{c}20-44 \\
\text { Ans }\end{array}$ & $\begin{array}{c}45-60 \\
\text { ans }\end{array}$ \\
\hline 61 hommes & 33 & 28 & 16 & 28 & 17 & $/$ \\
\hline 16 femmes & 14 & 2 & 5 & 2 & 9 & $/$ \\
\hline $\begin{array}{l}\text { Total : } \\
77 \text { répondan }\end{array}$ & 47 & 30 & 21 & 30 & 26 & $/$ \\
\hline
\end{tabular}

Nos données montrent que la majorité des paramédics hommes (33) et femmes (14) est âgée de 20-44 ans, qu'il s'agit d'une main d'œuvre à prédominance masculine, que les femmes âgées de 20-44 ans (9 soit 64,28 \%) sont davantage que les hommes (17 soit $51,51 \%$ ) à temps partiel. Notons que $100 \%$ des hommes et des femmes de 45-60 ans ont un contrat à temps complet, ce qui s'explique par leur ancienneté dans l'organisation et par leur volonté d'avoir un meilleur salaire.

\subsection{Les horaires des paramédics}

Nous avons évoqué plus haut les défis des horaires dans certaines professions de la santé, et les paramédics sont particulièrement touchés. Ils travaillent sur différents types 
d'horaires : des horaires de quarts journaliers de travail rémunéré à l'heure $(8,10,12$ heures) et des horaires de faction, notamment l'horaire 7/14. L'horaire 7/14 signifie que les paramédics sont de garde 7 jours sur 7 et demeurent au point de service ou à leur domicile, ce dernier devant alors être situé à 5 minutes au plus du point de service. Les 7 jours suivants ils sont en congé. Cela équivaut à dire qu'ils sont en devoir 168 heures sur une période de 14 jours et sont payés pour 80 heures. Le tableau 3 présente les horaires effectué par nos répondants.

\section{Tableau 3. Horaires des paramédics}

\begin{tabular}{|l|c|c|c|c|c|c|}
\hline & \multicolumn{2}{|c|}{$\begin{array}{c}\text { Horaires } \\
7 / 14\end{array}$} & \multicolumn{2}{c|}{ Sur appel } & \multicolumn{2}{c|}{$\begin{array}{c}\text { Horaires de } \\
8 \mathrm{~h}, 10 \mathrm{~h}, 12 \mathrm{~h}\end{array}$} \\
\hline \multicolumn{1}{|c|}{ Répondants } & $20 / 44$ ans & $45 / 60$ ans & $20 / 44$ ans & $45 / 60$ ans & $20 / 44$ ans & $45 / 60$ ans \\
\hline 61 hommes & 24 & 20 & 5 & & 4 & 8 \\
\hline 16 femmes & 7 & 2 & 2 & & 5 & 8 \\
\hline Total : 77 répondants & 31 & 22 & 7 & & 9 & 9 \\
\hline
\end{tabular}

Ce tableau montre que la majeure partie des répondants hommes et femmes travaille sur des horaires 7/14. Chez les 20-44 ans, 24 hommes (72,72\%) et 7 femmes $(50 \%)$ ont ce type d'horaires. En ce qui concerne la tranche des 45-60 ans, 20 hommes (71,42\%) et les 2 seules femmes dans la même tranche d'âge, effectuent également ce même type d'horaires. Aucune personne de plus de 45 ans ne travaille sur appel.

Nous interrogions également nos répondants sur leur satisfaction quant à leurs horaires de travail et nous avons vu que dans la tranche d'âge des 20-44 ans, 32 hommes $(96,97 \%)$ disent ne pas l'être, de même pour 11 femmes (78,57\%). En ce qui concerne les 45-60 ans, 15 hommes $(53,57 \%)$ ne le sont pas non plus. Les deux femmes de ce groupe d'âge ne le sont pas non plus. L'ensemble des répondants (17 soit $22.08 \%$ ), genres et âges confondus, qui travaillent sur des horaires à l'heure sont satisfaits de leur horaires. Ce sont les jeunes de 20-44 ans, hommes et femmes, qui présentent le plus haut taux d'insatisfaction.

\subsubsection{Impact des horaires sur le stress, le sommeil et l'irritabilité}

Nos répondants ont également fait état des effets des horaires sur le stress, le sommeil, l'irritabilité et l'appétit.

Tableau 4. Impact des horaires sur le stress, le sommeil, l’irritabilité, l'appétit

\begin{tabular}{|c|c|c|c|c|c|c|c|c|c|}
\hline & \multicolumn{4}{|c|}{ HOMMES } & \multicolumn{4}{|c|}{ FEMMES } & \multirow[b]{2}{*}{$\begin{array}{l}\text { Nombre de répondants } \\
\text { ayant répondu }\end{array}$} \\
\hline & $\begin{array}{l}\text { Oui } \\
\text { M44 }\end{array}$ & $\begin{array}{l}\text { Oui } \\
\text { P44 }\end{array}$ & $\begin{array}{l}\text { Non } \\
\text { M44 }\end{array}$ & $\begin{array}{l}\text { Nor } \\
\text { P44 }\end{array}$ & $\begin{array}{l}\text { Oui } \\
\text { M4 }\end{array}$ & $\begin{array}{l}\mathrm{Ou} \\
\mathrm{P} 4\end{array}$ & $\begin{array}{l}\text { Non } \\
\text { M44 }\end{array}$ & $\begin{array}{l}\text { Nor } \\
\text { P44 }\end{array}$ & \\
\hline Stress & 23 & 10 & 9 & 15 & 8 & 2 & 2 & 1 & $69 / 77$ \\
\hline Troubles du sommeil & 27 & 9 & 6 & 14 & 8 & 2 & 3 & / & $69 / 77$ \\
\hline Irritabilité & 13 & 14 & 10 & 9 & 5 & 2 & 2 & 1 & $55 / 77$ \\
\hline Troubles de l'appétit & 14 & 13 & 10 & 9 & 7 & 2 & 3 & 1 & $58 / 77$ \\
\hline
\end{tabular}

M44 signifie moins de 44 ans ou 20-44 ans; et P44 signifie plus de 44 ans, ou 45-60 ans.

Notons que toutes les personnes n'ont pas répondu. 
On voit que dans la catégorie des $20-44$ ans, 27 hommes $(81,81 \%)$ et 8 femmes $(72,72 \%)$ souffrent de troubles du sommeil. Le stress est également un irritant puisque 23 hommes $(71,87 \%)$ et 8 femmes $(80 \%)$ en souffrent. En ce qui concerne la catégorie des $45-60$ ans, l'irritabilité est signalée chez 14 hommes $(60,87 \%)$ et les troubles de l'appétit pour 13 hommes $(59,09 \%)$, alors que les deux femmes disent que les horaires de travail ont un impact sur le stress, le sommeil, l'irritabilité et l'appétit.

Nos résultats montrent que les horaires de travail ont des effets différents en fonction de l'âge et du genre. Cependant, il faut reconnaitre qu'il s'agit ici des perceptions des individus; nous n'avons bien sûr pas pu faire une étude médicale de la question. Malgré les difficultés liées aux horaires, aux répercussions de celles-ci sur la santé des employés, et aux difficultés quant à la conciliation travail-famille, à notre question «Votre profession, est-ce une vocation?», 61 répondants hommes et femmes de tous les âges disent que leur métier est une vocation; pour les autres, soit ils exerçaient auparavant une autre profession ou ils ont profité d'une opportunité. Les répondants expliquent avoir fait le choix du métier de paramédic afin d'aider les personnes en difficulté, attirées le côté urgence du métier qui fait monter l'adrénaline, le fait que ce ne soit pas un métier routinier et qu'il présente de nombreux défis à relever.

\subsubsection{Les conséquences des horaires sur la vie conjugale}

Tout d'abord, il faut savoir que les paramédics demeurent en attente d'affectation pendant 7 jours, soit à partir de la caserne (logement meublé au strict minimum, seulement une chambre pour 2 personnes) soit de leur domicile si celui-ci est situé à moins de cinq minutes de la caserne.En ce qui a trait à la vie conjugale, plusieurs répondants ont souligné les horaires comme pouvant être en partie responsables de frictions, de séparations, voire de divorces au sein des couples. Les propos ci-dessous permettent de voir l'effet à moyenlong terme de ces horaires et donc l'importance de prendre en compte la gestion des âges sur l'ensemble du parcours de vie. Notons que plusieurs paramédics sont en couple, mariés ou conjoints de fait avec des paramédics et travaillent dans cette même organisation.

Femme, 32 ans, conjoint de fait avec un paramédic, sans enfants, horaires de faction 7/14 : «C'est sûr que pendant un certain temps, tu te vois pas, on s'ennuie...on a comme eu notre vie de célibataire pendant tout ce temps-là et tout à coup pouf, tu te retrouves ensemble, c'est sûr que ça fait des conflits, cette situation-là. Dans le fond t'es comme un peu dans un état dépressif...il t'énerve un peu (rire)».

Homme, 52 ans, seul, 4 enfants, horaires de 8 heures : "Oui, bien quand j'étais sur un 7/14, j'ai eu la chance de me séparer deux fois (ironique). Parce que tu pars à toute heure de la nuit ou du jour faire des appels, ça fait que tu ne vois pas beaucoup tes enfants, t'as toujours une gardienne avec toi à la maison parce que ta femme travaille. Quand elle rentre, elle aimerait ça que tu restes avec elle, tu ne peux pas, toi tu repars, ça fait que la conciliation familletravail, c'est assez difficile».

Sur ce point, de manière générale, les hommes et les femmes de tous les âges soulignent la fragilité du couple en lien avec les horaires de travail. Plusieurs femmes paramédics mariées avec des paramédics de cette même organisation ajoutent la difficulté de vivre chacun de son côté pendant 7 jours et de se retrouver les 7 jours suivants 24 heures sur 24 . 
Nos répondants ont également souligné ce même type d'incidences, alors que nous posions la question de l'influence de ces horaires, en les invitant à aborder la question de la vie familiale et sociale. Pour illustration, voici quelques propos :

Femme, 31 ans, mariée, sans enfants, horaires de faction $7 / 14$ «Bien familiale, c'est comme je disais, même si je voudrais avoir des enfants, c'est pas possible avec cet horaire-là, il faut que je fasse un choix, de laisser mon travail ou bien de prendre un temps partiel dans le bas de la liste pour pouvoir me retrouver, on ne sait jamais, finalement sur appel, ou je n'ai pas d'enfants. (...) Dans le fond, ça l'empêche complètement là, la vie familiale».

Homme, 38 ans, marié, 1 enfant, horaire de faction $7 / 14$ «On est là, puis on n'est pas là, ma conjointe part à l'épicerie pour une demi-heure, puis je reste avec mon enfant qui a deux ans. Il faut vraiment que je me «calle» une gardienne ou je me «backe » avec une voisine, que si je pars sur un appel, qu'elle peut traverser....».

Homme, 41 ans, marié, 1 enfant, horaires à l'heure "On peut toujours demander une fête sur deux, on a le droit, mais en tant que temps partiel dans les premières années de carrière, on se doit de travailler toutes ces fêtes-là, les anniversaires....».

Pour les jeunes femmes de 20-44 ans ayant des enfants en bas âge ou prêtes à fonder un foyer, il est impensable de travailler dans ces conditions d'horaires. D'ailleurs, plusieurs d'entre elles qui possédaient un contrat à temps complet et qui étaient bien situées dans la liste d'ancienneté ont demandé à changer de statut d'emploi (en passant au temps partiel), ce qui leur a permis d'effectuer des horaires plus réguliers et de rentrer chez elles tous les soirs. D'autres, en âge de fonder un foyer, renonceront à avoir un enfant tant qu'elles ne seront pas titulaires de leur poste de travail, ce qui permettrait de travailler à temps partiel et de choisir ses horaires. D'autres encore songent à quitter leur emploi pour un emploi à heures fixes.

Les hommes de 20-44 ans énoncent plutôt des idées de logistique à mettre en place pour la garde des enfants. De manière générale, c'est plutôt du ressort de la conjointe «compréhensive» qui prend la totalité du congé parental lors de l'arrivée d'un enfant dans le foyer et qui travaillera à temps partiel par la suite. Ils évoquent également le fait que la famille, les parents, les voisins peuvent être mobilisés pour la garde des enfants en cas de difficultés. D'ailleurs, les jeunes hommes paramédics à temps partiel, occupent souvent un second emploi, le nombre insuffisant d'heures proposées par l'employeur principal ne permettant pas de vivre décemment. Nous n'avons pas relevé ceci chez les femmes dans les deux tranches d'âge, ce qui s'explique par le fait que l'homme est généralement vu comme le pourvoyeur principal.

Aucun homme de 45-60 ans n'envisage de travailler à temps partiel. Ils ne souhaitent pas travailler moins d'heures non plus car ils ne souhaitent pas voir leur salaire diminuer. Sur ce point le syndicat tente de négocier une mesure de retraite progressive sous forme de 4 jours travaillés, 5 jours payés. D'ailleurs, il faut noter que 13 hommes $(65,00 \%)$ dans la tranche d'âge 45-60 ans travaillant en horaires de faction disent apprécier ces horaires particuliers. Plusieurs raisons peuvent expliquer ceci; en effet, ces paramédics vivent à quelques minutes du point de service et en l'absence d'appels, peuvent vaquer à leurs occupations (faire du vélo, profiter de leur piscine, jardiner etc.) comme bon leur semble, 
chez eux. De plus, le secteur géographique dans lequel ils travaillent joue un rôle important, en ce sens que certains points de service ruraux enregistrent peu d'appels ( 1 à 2 maximum par jour) alors que dans d'autres endroits (près des villes) ils peuvent être appelés plus souvent. En outre, leurs enfants sont grands et autonomes ou ils n'en ont pas eu. Pour finir, pour plusieurs d'entre eux, le fait de ne travailler qu'une semaine sur deux permet de cumuler deux emplois en travaillant ailleurs pendant la semaine de congé ou encore de s'adonner à des loisirs.

Les répondants mettent en exergue la porosité des frontières entre la sphère du travail et celle de la vie familiale, autant pour les hommes que pour les femmes, mais nous remarquons toutefois que les préoccupations liées à la maternité, aux soins des enfants sont plus fréquentes chez les femmes. De plus, l'arrivée d'un enfant ne semble pas influencer la trajectoire professionnelle de nos répondants masculins alors que c'est le cas chez les femmes.

\subsubsection{L’imprévisibilité des horaires}

L'imprévisibilité des horaires est également problématique quant à la gestion du temps personnel et professionnel car cela réduit la vie sociale, ainsi que la stabilité de la vie familiale ou conjugale, comme l'indiquent nos répondants :

Femme, 31 ans, marié, sans enfants, horaires à l'heure : «Euh, les horaires...Comme ça, d"être sur appel toujours, de ne pas pouvoir planifier ou travailler de nuit, ce n'est pas toujours facile...Combiner une nuit...Là comme cette nuit je travaille de nuit, peut-être que demain je vais être appelée pour rentrer de soir, jeudi de jour, de bonne heure, c'est difficile de trouver une stabilité»».

Homme, 34 ans, marié, 1 enfant, horaires de faction 7/14: «Je pense que le gros élément négatif, c'est surtout ça, les horaires où ce qu'on ne peut pas rien planifier, on ne peut pas avoir de famille non plus, on ne peut pas avoir de gardienne $24 \mathrm{~h} / 24$ disponible, quand on a des horaires comme ça, tu sais on ne peut pas avoir de loisirs, si on veut s'inscrire aller jouer au hockey une fois par semaine, bien une semaine sur deux, on ne peut pas y aller, toutes les activités c'est ça, on ne peut pas avoir, tu sais un horaire comme tout le monde».

Ainsi, selon nos répondants, les horaires de faction et de nuit ne permettent pas aux individus de s'organiser et ce, tant dans la vie professionnelle que dans la vie personnelle.

\subsubsection{L'âge et les horaires atypiques}

Si les horaires de faction sont difficiles lorsqu'on est jeune, en raison des conséquences sur la vie familiale et sociale, il semble que plusieurs travailleurs pensent que ces horaires peuvent être encore plus difficiles avec l'avancée en âge, comme l'indiquent ces extraits :

Homme, 32 ans, conjoint de fait, 1 enfant, horaires à l'heure: "Quand on vieillit on s'attend à une meilleure qualité de vie et si cette meilleure qualité de vie-là ne vient pas à cause du travail, je peux comprendre qu'il y en a qui ont des frustrations par rapport à ça».

Femme, 50 ans, mariée, 2 enfants, horaires de faction 7/14 : «Il faudrait que je « tofferais » selon eux jusqu'à soixante ans, je ne suis pas sûre encore dix ans, tu sais avoir la force physiquement... En vieillissant je le vois encore plus, parce que tu sais, en tout cas, plus 
jeune, il me semble j'allais dormir une couple d'heures, tu sais je revenais d'un appel, j'étais capable de redormir, mais là on dirait que depuis quelques années, en vieillissant, je ne sais pas pourquoi, j'ai bien de la difficulté à dormir le jour, fait que je pense qu'un moment donné ça atteint la santé».

Homme, 51 ans, 3 enfants, horaires de faction 7/14: «Professionnellement si je suis capable de me rendre dans 5 ans, je vais être content. Parce que disons que moi j'ai les genoux finis, j'ai des prothèses, dans le genou droit j'ai une prothèse complète. Il ne faut pas se le cacher, j'ai juste 51 ans...».

Les difficultés physiques perçues par les hommes et femmes de tous les âges peuvent constituer un frein pour mener à bien ou prolonger sa carrière.

\subsection{Les pratiques d'ARTT du point de vue de la conciliation travail-famille}

Comme les préoccupations pour la rétention de main-d'œuvre sont importantes dans ce secteur, nous avons interrogé les répondants sur les pratiques d'ARTT (aménagement et réduction du temps de travail) qui pourraient les intéresser du point de vue de la conciliation travail-famille et avons mis en évidence les choix les plus intéressants et les moins intéressants pour eux. Nous avons établi une série de tableaux détaillés sur ceci, mais résumons les grandes lignes, en raison des contraintes d'espace.

La retraite avec un emploi à temps partiel constitue une option jugée très intéressante à intéressante par 29 hommes, soit 87,88\% dans la tranche d'âge de 20-44 ans; de même pour 27 hommes $(81,82 \%)$ concernant la retraite progressive et les horaires flexibles. Dans la même tranche d'âge, 13 femmes (92,86\%) qualifient de très intéressant à intéressant la retraite progressive, la retraite et emploi à temps partiel, les jours supplémentaires de congé et les vacances annuelles plus longues. En comparant les mesures choisies par les répondants de 20-44 ans, nous observons qu'hormis la retraite progressive et la retraite et emploi à temps partiel, les hommes marquent un intérêt certain pour les horaires flexibles alors que les femmes marquent plutôt leur préférence sur les jours supplémentaires de congés et les vacances annuelles plus longues. Elles veulent se dégager des blocs de temps.Dans la tranche d'âge des 45-60 ans, 28 hommes (100\%) disent être très intéressés par la retraite progressive, 26 hommes $(92,86 \%)$ par les jours supplémentaires de congé et 25 hommes (89,29\%) par les vacances annuelles plus longues.Les 2 femmes dans la tranche d'âge des 45-60 ans, disent être très intéressées par la retraite progressive, la retraite et emploi à temps partiel, la semaine réduite à 4 jours et vacances annuelles plus longues. Unanimement, les intérêts pour la retraite progressive convergent pour les répondants des deux sexes dans la tranche d'âge des 45-60 ans; ceci serait donc une option intéressante pour la gestion des âges en fin de carrière.

Diverses stratégies sont utilisées par nos répondants pour concilier la vie professionnelle et personnelle. Effectivement comme nous le disions plus haut, plusieurs femmes qui étaient employées à temps plein ont opté pour un temps partiel afin de pouvoir mieux articuler la dimension professionnelle et privée, d'autres hommes et femmes ont pu compter sur la compréhension de leur conjoint, d'autres encore se servent de leur banque de congés payés et maladie, de congés sans solde pour répondre aux imprévus. Aussi, 
plusieurs ont des stratégies d'échanges ou de verbalisation pour passer d'une sphère à l'autre. Les hommes plus âgés se sont organisés différemment en vivant à 5 minutes du point de service.

Homme, 52 ans, conjoint de fait, sans enfants, horaire de faction 7/14 : «Je me suis poussé en région, là l'horaire 7/14 moi je le vis agréablement bien, donc j'ai même quitté la ville pour rester sur ce genre d'horaire-là qui me convient très bien. Je suis à mon domicile, puis quand on a des appels on se rend au poste, les paramédics demeurent tous à plus ou moins 1 $\mathrm{km}$ du poste d'ambulance, fait qu'on est tous proche».

Femme, 31 ans, mariée, 1 enfant (en congé parental), horaires à l'heure dont 7/14 : «Je viens d'avoir un enfant alors j'ai abandonné mon temps complet 7/14 pour un temps partiel sur un chiffre à l'heure, j'avais l'ancienneté pour le faire. C'est sûr que là ça joue sur mon salaire, mais non, je n'en referai pas de 7/14. Si j'ai à retravailler sur du 7/14, je vais prendre des congés, je vais être malade, je vais prendre du sans solde si on m'en donne, je n'en ferai plus, c'est sûr, ça c'est clair dans ma tête»

Cependant il faut noter que l'utilisation de ces stratégies est restreinte. En effet, seuls les employés possédant un contrat à temps plein peuvent passer à temps partiel (selon la localité il peut tout de même y avoir des horaires de faction) et cela en fonction de leur position sur les listes d'ancienneté. Il n'est également pas toujours facile, lorsqu'il s'agit de couples avec ou sans enfants, de déménager (l'un ou l'autre des parents sera toujours éloigné du domicile par rapport à son travail). La recherche d'une nouvelle gardienne est également problématique (planification peu prévisible) avec les horaires atypiques effectuées par les paramédics.

Il est également important de préciser que les congés sans solde, les congés de maladie, les congés payés, les congés de maternité (les femmes paramédics bénéficient du retrait préventif (grossesse et allaitement) et de parentalité sont les seules mesures offertes. Ces mesures sont beaucoup utilisées afin d'obtenir une meilleure articulation de la vie professionnelle et personnelle. La plupart des jeunes femmes indiquent qu'elles cherchent à concilier le travail avec leur rôle de mère. Quant aux hommes (45-60 ans) ils s'organisent plutôt pour pouvoir rester chez eux à attendre les appels.

\section{Conclusion}

Nous avons pu observer que les conséquences des horaires sont particulièrement difficiles pour les jeunes hommes et femmes ayant de jeunes enfants, mais ces difficultés posent aussi problème pour la rétention. Les difficultés persistent pour les femmes plus âgées, puisqu'elles continuent d'assumer les responsabilités familiales, alors que les hommes sont toujours davantage investis dans le milieu de travail que dans la famille.

En ce qui a trait aux solutions, pour la conciliation comme pour la rétention, soit les mesures d'aménagement du temps de travail, il est vrai que certaines mesures sont difficilement envisageables dans ce type d'emploi (horaires flexibles, journée de travail plus courtes, etc.). Ainsi, que ce soient les jeunes hommes ou les hommes plus âgés, tous montrent un intérêt pour les mesures en lien avec la retraite progressive, ainsi que la 
retraite et temps partiel. Cependant, les plus âgés ne sont pas prêts à envisager la retraite progressive, s'ils doivent perdre en salaire. Les femmes jeunes et plus âgées soulignent qu'outre les mesures «de retraite progressive et de retraite et temps partiel», les mesures «de jours supplémentaires de congé, de vacances annuelles plus longues, de semaines comprimés (les plus âgés)» pourraient les aider à mieux articuler le travail et la famille tout au long du parcours de vie. Il semble donc urgent de trouver une solution pour tous les paramédics car ils vivent des difficultés non négligeables sur le plan des horaires. Ceci pourrait permettre une meilleure rétention et fidélisation des paramédics dans l'organisation et dans le métier.

Bien qu'elle doive donc être considérée comme exploratoire, en raison du nombre limité de répondants sur le plan statistique, cette recherche présente un secteur peu ou pas étudié à ce jour, ce qui constitue un apport important, malgré la limite du nombre. Cette recherche illustre bien les difficultés particulières du milieu des paramédics et invite à développer des mesures répondant aux besoins particuliers de chacun des groupes (âge et genre), afin d'assurer une bonne articulation vie professionnelle/vie personnelle (Boivin, 2009). Ainsi il semblerait possible d'éviter certains absentéismes, des départs prématurés tout en conciliant les besoins des paramédics et ceux des patients en préservant la continuité et la qualité des soins, c'est-à-dire la stabilité des effectifs.

\section{Références}

Allen, T. D., Herst, D. E., Bruck, C. S. \& Sutton, M. (2000). Consequences associated with work-to-family conflict: A review and agenda for future research. Journal of Occupational HealthPsychology, 5(2), 278-308.

Anact (2012). Pénibilité et usure professionnelle; comment prévenir ? http://www.anact.fr/web/dossiers/sante-au-

travail/accord_penibilite?p_thingIdToShow=20715571 (page consultée le 9 avril 2012).

Boivin, J., Lutumba Ntetu, A.,Poirier, D. (2009). Facteurs explicatifs de la non-rétention du personnel infirmier : étude de la situation au Service régional Info-Santé et Info-Social du Saguenay Lac-St-Jean, L'infirmière clinicienne, Vol 6, n¹, 1-10.

Association professionnelle des paramédics du Québec, (s.d.). Qui sont les paramédics au Québec? Récupérée le 03 janvier 2011 à partir de http://www.paramedicduquebec.org/paramedics/qui-sont-les-paramedics/

Chevandier, C. (2009). Vocation professionnelle : un concept efficient pour le XXème siècle? Annales de Bretagne et des Pays de l'Ouest, n 116/3, p.95-108 Rennes : Presses Universitaires de Rennes.

Cloutier, E., E. Ledoux, H. David (2007). L'importance de l'organisation du travail comme soutien à la transmission des savoirs Dans D.-G. Tremblay (dir.), D'une culture de la retraite à un nouveau management des âges et des temps sociaux. Québec : Presses de l’Université du Québec. 294 p. 
Corbeil, P. Plamondon, A. Normand, N. Teasdale, A. Tremblay, M. Simoneau, (2010) Mesure de l'exposition du technicien ambulancier paramédic aux facteurs de risque de troubles musculo-squelettiques. 1p. Québec: IRSST/Université Laval.

Confédération des Syndicats Nationaux. (2006). Délais d'intervention des ambulanciersparamédics : des milliers de citoyens des régions du Québec réclament des actions immédiates.

Récupérée le 10 octobre 2011 à partir de http://www.csn.qc.ca/web/csn/communique//ap/Comm14-06-06a.xml

David, H. (2007). Quand la gestion des âges est inapplicable : la précarité d'emploi d'infirmières en soutien à domicile. Dans D.-G. Tremblay (dir.), D'une culture de la retraite à un nouveau management des âges et des temps sociaux. Québec : Presses de l’Université du Québec. 294 p.

Desjardins, S. (2004). Le sommeil chez les travailleurs de nuit, Ordre des psychologues du Québec, Psychologie Québec p. 32-35.

Duxbury, L. E., Higgins, C.A, \& Lee, C. (1994). Work-Family Conflict. A Comparison by Gender, Family Type and Perceived Control, Journal of Family Issues, 15(3), 449-466.

Fusulier, B. Giraldo, S. \& Laloy, D. (2008). L'entreprise et l'articulation travail/famille. Transformations sociétales, supports institutionnels et médiation organisationnelle. UCL Presses Universitaires de Louvain, 199 p.

Fusulier, B., Laloy, D., \& Sanchez E. (2009). Être au service et articuler travail/famille, Informations sociales, $\mathrm{n}^{\circ} 154$ p.22-30.

Gavard-Perret. M.L, Gotteland, D. Haon, C. Jolibert, A. (2008). Méthodologie de la recherche : Réussir son mémoire ou sa thèse en sciences de la gestion. Pearson Education, $383 \mathrm{p}$.

Gavrancic, A., Courcy, F., Proulx, J. (2009). Comment superviser une équipe de travail diversifiée? Gestion, vol 34, n 2

Lazzari-Dodeler, N. \& Tremblay, D.-G. (2010). Analyse de mesures et services en faveur de la conciliation travail-vie personnelle. Note de recherche no 2011-1 de 1'ARUC sur la gestion des âges et des temps sociaux. Montréal : ARUC-GATS. www.teluq.uqam.ca/arucgats

MacBride, J.-L., \& Bachman, K. (1999). L'équilibre travail-vie personnelle pose-t-il toujours problème aux Canadiens et à leurs employeurs ?Et comment!, Ottawa: Conference Board du Canada 
Marbot, N. (2007). La gestion des âges et l'équité entre les générations, Retraite et société, $\mathrm{n}^{\circ} 51$, p. 103-125.

Ministère de la Santé et des Services Sociaux. (2002). Planification de la main d'œuvre dans les services préhospitaliers d'urgence, Québec : Ministère de la santé et des services sociaux. 81p.

Morissette, R. Schellenberg, G. \& Silver, C. (2004). Inciter les travailleurs âgés à rester en poste. L'emploi et le revenu en perspective, Statistique Canada, vol 16, $n^{\circ} 4$.

Morin, C.-M. (2009).Vaincre les ennemis du sommeil, Montréal, Les Éditions de l'Homme, 288 p.

Morin, E. (2008). Sens du travail, santé mentale et engagement organisationnel. Santé psychologique, Études er recherches, Rapport R-543. Récupéré le 12 décembre 2010 à partir de http://www.irsst.qc.ca/media/documents/pubirsst/r-543.pdf

Pailhé, A., \& Solaz, A. (2010). Concilier, organiser, renoncer : quel genre d'arrangements? Travail, genre et sociétés $\mathrm{n}^{\circ} 24$, p. 29-46

Paris, H. (1989a). Les programmes d'aide aux employés qui ont des obligations familiales, Ottawa, rapport $\mathrm{n}^{\circ} 43$, Ottawa, Conference Board du Canada.

Pichonnaz, L. (2011). La difficile reconnaissance des compétences des ambulanciers : entre care, cure et force physique, Travailler, ${ }^{\circ} 26$, p. 17-33.

Presser, H.-B. (2004). Économie de 24 heures sur 24, structuration du temps et vie familiale, Âges, générations et contrat social, Les cahiers de l'Ined, n 153, p. 245-261.

Services Canada. (2009). 3234 - Ambulancier et autre personnel paramédical, textes de la profession. Récupérée le 13 février 2011 à partir de

http://www.servicecanada.gc.ca/fra/qc/emploi_avenir/statistiques/3234.shtml

Shields, M. (2000). Long Working Hours and Health. Perspectives on Labour and Income, Statistique Canada, vol. 12, pp. 49-56.

Thomas, D.-R. (2006). A general inductive approach for analyzing qualitative evaluation data. American Journal of Evaluation, 27(2), p. 237-246.

Tremblay, D.-G. (2007, dir.). D'une culture de la retraite à un nouveau management des âges et des temps sociaux. Québec : Presses de l'université du Québec, 281 p.

Tremblay, D.-G. (2008). Vers une articulation des temps sociaux tout au long de la vie; l'aménagement et la réduction du temps de travail. Montréal : Ed. Saint-Martin., 468 p.

Tremblay, D.-G. (2010). D'une culture de retraite vers de nouvelles fins de carrière? Montréal : Editions St Martin et Décarie Éditeur.143 p. 
Tremblay, D.-G. (2012a). Conciliation emploi-famille et temps sociaux, 3e Edition, Québec : Presses de l'université du Québec. 406 p.

Tremblay, D.-G (2012b). Concilier emploi et famille. Le rôle du soutien organisationnel au coeur de trois professions (infirmières, travailleuses sociales et policiers). Québec: Presses de l'université du Québec.

Tremblay, D.-G., Fusulier, B., \& di Loreto, M. (2009). Le soutien organisationnel à l'égard des carrières : le travail social, un milieu de travail (peu) favorable à la conciliation emploifamille ? Revue multidisciplinaire sur l'emploi, le syndicalisme et le travail. Vol. $4, \mathrm{n}^{\circ} 1$, pp. 27-44.

Tremblay, D.-G et Genin, E. (2010). Aging, economic insecurity and employment: Which measures would encourage older workers to stay longer in the labour market? Studies in Social Justice, vol.3, no 2 , http://ojs.uwindsor.ca/ojs/leddy/index.php/SSJ/article/view/2878/2348

Tremblay, D.-G. \& Larivière, M. (2010). L'articulation emploi-famille dans le secteur infirmier au Québec. Une conciliation possible? Éthique publique. Vol. 11, n 2, 43-50.

Uriarte-Landa, J. et Hébert, B.-P (2009). La conciliation travail-vie personnelle des travailleurs âgés, L'emploi et le revenu en perspective, Vol 10, nº10, p.19-32.

Weber, M. (1919). Le savant et le politique, Paris, Union Générale d'Éditions, 1963, 186 p. Collection : Le Monde en 10-18. Une édition électronique réalisée à partir du livre de Max Weber Récupérée le 05 février 2011 à partir de

http://classiques.uqac.ca/classiques/Weber/savant_politique/Le_savant.pdf 